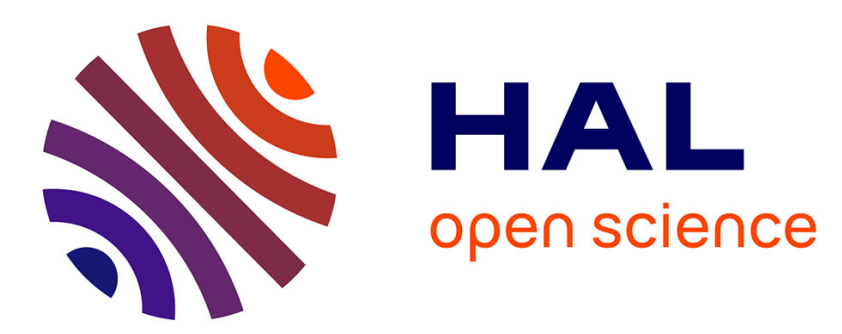

\title{
TDMA based MAC Transmission Schedules in Multihop Grid Ad hoc Underwater Acoustic Networks
}

\author{
Said Lmai, Mandar Chitre, Christophe Laot, Sébastien Houcke
}

\section{To cite this version:}

Said Lmai, Mandar Chitre, Christophe Laot, Sébastien Houcke. TDMA based MAC Transmission Schedules in Multihop Grid Ad hoc Underwater Acoustic Networks. UComms 2014: underwater communications conference, Sep 2014, Sestri Levante, Italy. 10.1109/UComms.2014.7017131 . hal01188839

\section{HAL Id: hal-01188839 \\ https://hal.science/hal-01188839}

Submitted on 11 Jun 2021

HAL is a multi-disciplinary open access archive for the deposit and dissemination of scientific research documents, whether they are published or not. The documents may come from teaching and research institutions in France or abroad, or from public or private research centers.
L'archive ouverte pluridisciplinaire HAL, est destinée au dépôt et à la diffusion de documents scientifiques de niveau recherche, publiés ou non, émanant des établissements d'enseignement et de recherche français ou étrangers, des laboratoires publics ou privés. 


\title{
TDMA-based MAC transmission schedules in multihop grid ad hoc underwater acoustic networks
}

\author{
Said Lmai ${ }^{1}$, Mandar Chitre ${ }^{2}$, Christophe Laot $^{1}$, and Sebastien Houcke ${ }^{1}$ \\ ${ }^{1}$ TELECOM Bretagne, UMR CNRS 6285 Lab-STICC, Université Européenne de Bretagne, Technopôle \\ Brest-Iroise CS83818- 29238 Brest Cedex 3, France \\ ${ }^{2}$ Electrical and Computer Engineering Department and Acoustic Research Laboratory (ARL), National University \\ of Singapore, Singapore 119227, Singapore
}

\begin{abstract}
Development and deployment of underwater acoustic (UWA) sensor networks have emerged as an efficient solution for many applications related to marine environment e.g., scientific, industrial, and military operations. The main tasks to execute are remote sensing and monitoring. Grid topology with multihop relaying is very useful, since it allows a wide area coverage as well as long distance data transmission. In this paper, we investigate architectures where sensing and monitoring data are forwarded over multiple lines independently. We are interested in transmission schedules which maximize network throughput. We prove that an optimal schedule is necessarily per-node fair. We also derive the upper bound. Furthermore, we present a low-complexity algorithm to find schedules achieving the upper bound, regardless of the size of the network.
\end{abstract}

\section{INTRODUCTION}

$\mathbf{T}$ HE primary applications of underwater acoustic (UWA) sensor networks are in scientific missions to observe the environment, industrial missions to monitor and manage commercial activities, and military missions to monitor and secure sensitive areas [1]. When the area involved is large, multiline grid topologies with multihop relaying are relevant architectures to consider, particularly for high-rate communication services. Multiline grid topology consists of several parallel vertical lines with regularly placed nodes. As in all sharedmedium networks, medium access control (MAC) protocol is necessary to regulate and coordinate UWA channel access. As the propagation delays in UWA networks are naturally large, traditional TDMA (time division multiple access) suffers from low performance due to the long guard time required. However, we use a version of TDMA where packet transmissions can overlap without colliding at a receiver, and this allows us to use the propagation delay more effectively. Contentionbased MAC protocols are usually more energy-consuming than their TDMA counterparts [2]. Transmission schedules that highly exploit large propagation delays allow higher network throughput, or comparably channel utilization, to be achieved. In section III of this paper, we prove that an optimal periodic schedule in a regular multiline grid network with multihop relaying, is necessarily per-node fair. The considered network is segmented into several partially-overlapping collision domains with unicast traffic. Furthermore, we derive the upper bound on network throughput. In section IV, we propose schedules to achieve the upper bound and present a computationally efficient algorithm for developing such schedules. The main intent is to allow as many simultaneous transmissions as possible, and to limit the interference, as much as is feasible, to unintended nodes.

The idea of taking advantage of large propagation delays has been considered in many studies (e.g. [3]-[6]). However, the performance in terms of normalized network throughput does not exceed 1, at best, regardless of the network topology adopted. Studies have been made on linear topology in multihop networking, although most of the interest was on physical link capability. The analysis in [7] takes into account interhop interference and shows achievable information rates versus per-node power. Nevertheless, no network-oriented performance is explored. In [8], multihop linear topology is explored under fair access criterion for all nodes. In our paper, message duration is set to one hop propagation delay. In such a context, authors in [8] derive a tight upper bound in terms of overall network utilization. However, we derive optimal schedules achieving a tighter upper bound. In [9], the authors prove that, within one collision domain, $N / 2$ is the maximum achievable network throughput in $N$-node network, and identify some geometries where developed schedules achieve this upper bound. In this paper, we use the valuable results in [9] to conduct a study with sharper focus. We concentrate our study on a multiline grid topology and we conduct an indepth analysis to explore how advantageous large propagation delays are. Section II describes the general context and system model and section $\mathrm{V}$ concludes the paper.

\section{CONTEXT AND SYSTEM MODEL}

In a nonzero propagation delay environment, we consider a regular $N$-node network with multiline grid topology where each node is identified by $i$ s.t. $i \in \mathbb{N}^{*}$ and $1 \leq i \leq N$. Let $\eta_{l i} \geq 1$ denote the number of independent node lines in the network. Messages originating from nodes $1,2, \cdots, \eta_{l i}$ are relayed hop by hop until they reach final destination nodes $N-\eta_{l i}+1, N-\eta_{l i}+2, \cdots, N$, respectively. An illustration of this architecture is given in Fig. 1. Let $\mathbf{r}_{i}$ be the position vector of node $i$ in 3-D Euclidean space. The propagation delays between every pair of nodes may be expressed using a delay matrix [9] denoted by $\mathbf{D}$. Time is assumed to be slotted.

$$
D_{i j}=\frac{\left|\mathbf{r}_{i}-\mathbf{r}_{j}\right|}{c \tau}, \quad 1 \leq i, j \leq N
$$




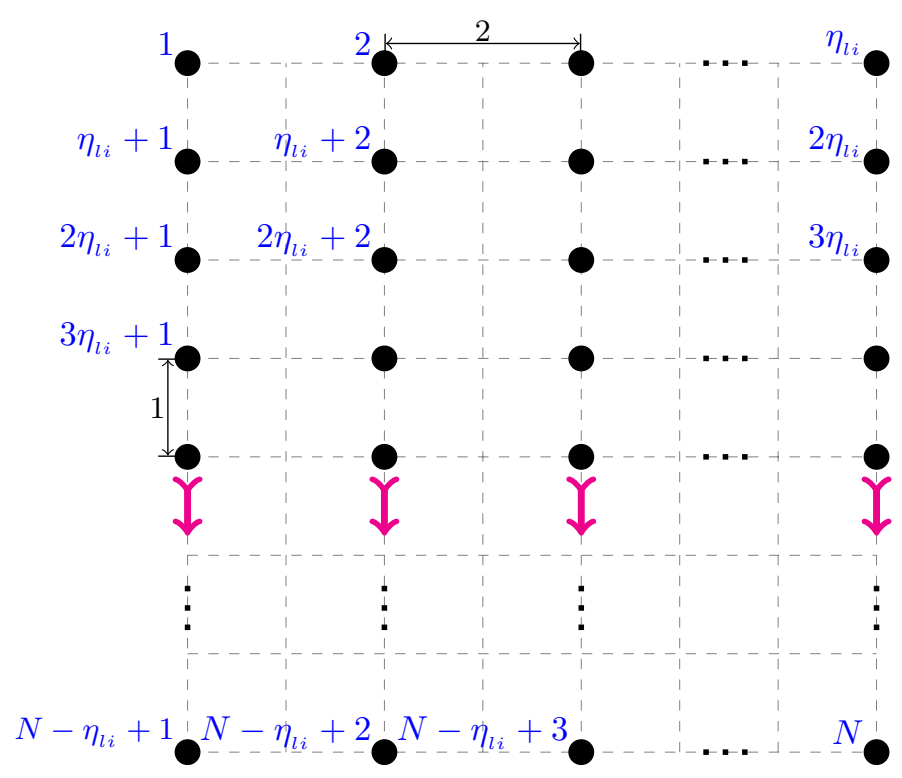

Fig. 1. Overview of the regular multiline grid $N$-node network.

where $c$ is the signal propagation speed and $\tau$ is the length of one time slot. Hence, the entries of $\mathbf{D}$ are nonnegative real numbers. Furthermore, we consider in this paper a network with unit spacing between every pair of neighboring nodes on the same line. Indeed, for the sake of analytical simplicity, we have also assumed that $\tau$ is set to the propagation delay between two neighboring nodes on the same line. The distance separating two adjacent node lines is 2 . The geometry of the network is fully described by the delay matrix. $\mathbf{D}$ is symmetric i.e., $D_{i j}=D_{j i}$, since $|i-j|=|j-i|$, and it has an all-zero diagonal i.e., $D_{i i}=0$.

In wireless radio networks, the interference range is often considered to be approximately twice the transmission range [10], [11]. In UWA environment, we assume the same proportions. We set the transmission range of each node to $g=1$. Actually, a single collision domain may be defined as the set of neighboring nodes with a shared-medium along which a transmission propagates, and where the resulting interferences terminate. Thus, a single collision domain is identified with respect to a certain node. We then have $N$ partially-overlapping collision domains. With regards to packet delivery, unicast traffic is used i.e., a message is sent from a single source node to a single destination node. Except for the source and destination nodes, a message is considered as an interference at all nodes that it reaches. All nodes are operating in half-duplex mode i.e., a node cannot simultaneously transmit and receive. Assuming the physical link to be reliable (error free) with constant data rate $\nu$, the loss of a message is due only to collision. A collision is said to occur at a certain node if two or more messages overlap in time. A successful transmission refers to a transmission that results in a successful reception of the message at the destination node. The normalized network throughput $Y$ is the total number of information bits successfully received by all nodes in the network per unit time, normalized by the link data rate $\nu$.

Provided that the message duration is equal to $\tau$, we define a transmission schedule $\mathbf{S}$ as the matrix that determines when each node transmits and receives messages [9]. The entries of $\mathbf{S}$ correspond to the different scenarios below.

- $S_{i t}=S_{i, t}=j>0$ indicates that node $i$ transmits a message to node $j$ at time slot $t$.

- $S_{j t}=S_{j, t}=-i<0$ implies that node $j$ receives a message from node $i$ during the time slot $t$.

- In all other cases, node $i$ is designated as an idle node during time slot $t$, which is represented by $S_{i t}=S_{i, t}=0$. If $S_{i, t+T}=S_{i t} \forall i, t$, this indicates that the schedule is repeating with a period $T$. It is said to be periodic with period $T$ and may be depicted using an $N \times T$ matrix $\mathbf{S}^{(T)}$ where

$$
S_{i t}=S_{i, t(\bmod T)}^{(T)} \text {. }
$$

Therefore, node $i$ transmits a message to node $j$ during time slot $t$ only if node $j$ is able to successfully receive the message during time slot $t+D_{i j}$ i.e.,

$$
S_{i t}=j \quad \Leftrightarrow \quad S_{j, t+D_{i j}}=-i \quad \forall i \neq j, D_{i j} \leq g .
$$

Furthermore, to ensure the successful reception at time slot $t$ of a transmitted message, it is required that no other nodes transmit messages that arrive at node $j$ during $t$. Thus,

$$
S_{j t}=-i \quad \Rightarrow \quad S_{k, t-D_{j k}} \leq 0 \quad \forall k \neq i, D_{j k} \leq 2 g .
$$

From $\mathbf{S}^{(T)}$, we can calculate the average network throughput considering the number of receptions in $\mathbf{S}^{(T)}$ :

$$
Y=\frac{1}{T} \sum_{t=0}^{T-1} \sum_{i=1}^{N} \mathbb{1}\left(S_{i t}^{(T)}<0\right),
$$

where $\mathbb{1}(E)$ is the indicator function of the event $E$, with value of 1 if $E$ is true and 0 otherwise. As introduced in [9], when a schedule $\mathbf{S}^{(T)}$ provides the same number of transmission opportunities to all nodes, $\mathbf{S}^{(T)}$ is said to be per-node fair i.e.,

$$
\sum_{t=0}^{T-1} \mathbb{1}\left(S_{i t}^{(T)}>0\right)=\text { constant }>0 \quad \forall i .
$$

Note that the final destination nodes are not affected by this fairness characteristic, since they either receive or remain idle at any time.

\section{Achievable throughpUt IN REgUlar MUltiline GRID $N$-NODE NETWORK}

We define an optimal transmission schedule as being the schedule that maximizes the network throughput. We shall start by showing an important feature of such a schedule.

Theorem 1. An optimal periodic transmission schedule in a regular multiline grid $N$-node network, with multihop relaying, is necessarily per-node fair.

Proof: Since every network has an optimal schedule that is periodic [9], we are interested in schedules $\mathbf{S}^{(T)}$ with period $T$. Assume that there is a transmission schedule $\mathbf{S}^{(T)}$ with at least two nodes $\bar{i}$ and $\bar{j}$ on the same line such that $\sum_{t=0}^{T-1} \mathbb{1}\left(S_{\bar{i} t}^{(T)}>0\right)>\sum_{t=0}^{T-1} \mathbb{1}\left(S_{\bar{j} t}^{(T)}>0\right)$ i.e., $\bar{i}$ transmits more often than $\bar{j}$. Let us consider nodes $i$ and $j$ where

$$
D_{i j}=\min _{\bar{i}, \bar{j}} \quad D_{\bar{i} \bar{j}} .
$$

Accordingly, $i$ and $j$ are neighboring nodes.

Case1: $i<j$ i.e., $j=i+\eta_{l i}$ 


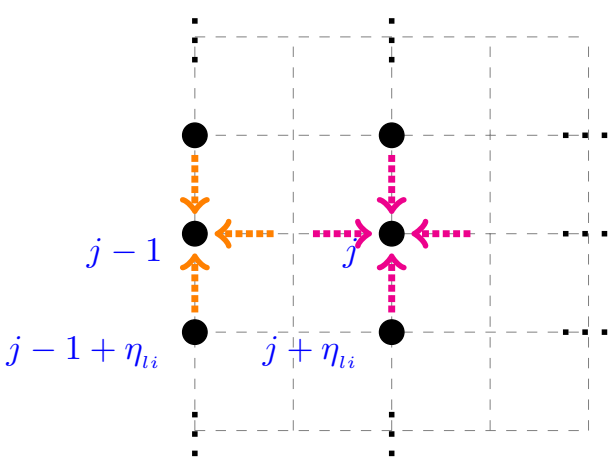

Fig. 2. Overview of interference impact.

A node $\bar{i}$ has, at most, three interfering neighbors if $\bar{i}$ belongs to an edge line, or four interfering neighbors if it belongs to an inner line (e.g., nodes $j-1$ and $j$ in Fig. 2 respectively). Node $j$ is not the first node on the line since it receives from $i(<j)$. Then $j$ is impacted by interference from three or four surrounding nodes. Note that Fig. 2 illustrates the case where $j$ has four interfering neighbors. Node $j+\eta_{l i}$ is similarly impacted by interference from, at most, four surrounding neighbors. However, over one period $T, i$ transmits $n_{i T}=\sum_{t=0}^{T-1} \mathbb{1}\left(S_{i t}^{(T)}>0\right)$ messages i.e., $j$ receives $n_{i T}$ messages, while $j+\eta_{l i}$ receives only $n_{j T}<n_{i T}$ messages. This contradicts the objective of any strategy aiming to maximize the network throughput in such a regular structure.

Case2: $i>j$ i.e., $i=j+\eta_{l i}$

Over one period $T$, node $i$ receives from node $j$ exactly $n_{j T}$ messages, while $i$ transmits $n_{i T}>n_{j T}$ messages. This indicates that $i$ transmits more messages than it has received from its downstream neighbor, an action which contradicts the multihop relaying concept adopted in the network.

Alternatively, nodes $\bar{i}$ and $\bar{j}$ could belong to two separate lines $L 1$ and $L 2$. If over $L 1$ or $L 2$, there exist nodes that do not transmit the same number of messages over one period $T$, the above demonstration is valid. Otherwise, we have two lines where all nodes transmit the same count of messages on each line. However, the total number of messages on $L 1$ and $L 2$ are different. Consider the most unfavorable case where $L 1$ is an edge line while $L 2$ is inner line. In fact, the key factor in maximizing the throughput is planning the arrival of the maximum number of interferences at the time slot that will be used by the node for transmitting. In Fig. 2, one will observe that managing three interferences, like those affecting node $j-1$, or four interferences, like those affecting node $j$, may be performed in exactly the same way. Indeed, the node cannot receive in the presence of one or several interferences. Given the regular geometry of the network, any strategy adopted to maximize throughput on a single line, should act identically on all other lines. The contexts where $L 1$ and $L 2$ are both edge lines or inner lines are more straightforward. Therefore, $L 1$ and $L 2$ could not be carrying disparate amount of data.

Knowing that an optimal periodic transmission schedule with multihop relaying is necessarily per-node fair, we derive the upper bound on network throughput.
Theorem 2. In a regular multiline grid $N$-node network with multihop relaying, the network throughput is upper bounded by $\left(N-\eta_{l i}\right) / 2$.

Proof: Consider a regular multiline grid $N$-node network where $N \geq 2$ and $\eta_{l i} \geq 1$. Each line forwards independently its own messages toward the final destination node at the network end. We have $N \geq 2 \eta_{l i}$. Let $\mathbf{S}^{(T)}$ be an optimal transmission schedule. According to theorem $1, \mathbf{S}^{(T)}$ is pernode fair. Thus, every node $i$, where $1 \leq i \leq N-\eta_{l i}$ transmits at least once during $T$ time slots. Therefore, among the $N T$ entries of $\mathbf{S}^{(T)}$, there exist at least $N-\eta_{l i}$ positive entries and $N-\eta_{l i}$ negative entries $\forall T$. The $\eta_{l i}$ respective final destination nodes of relayed traffic on each line, are $N-\eta_{l i}+1, N-\eta_{l i}+2, \cdots, N$. Each of these nodes receives at least $\left(N / \eta_{l i}\right)-1$ messages over one period of $T$ time slots. Let us consider a final destination node $j$, where $N-\eta_{l i}+1 \leq j \leq N$. Node $j-2 \eta_{l i}$ has transmitted at least one message to its neighbor $j-\eta_{l i}$ at time slot $t$. Due to the half-duplex constraint, node $j$ remains idle during time slot $t+2$ if node $j-\eta_{l i}$ transmits also at $t$, or during time slot $t+1$ if node $j-\eta_{l i}$ is not transmitting at $t$. Hence, there are $\eta_{l i}$ idle entries in $\mathbf{S}^{(T)} \forall T$.

Now, let us assume $T=1$ i.e., $\mathbf{S}^{(T)}$ is a column vector $(N \times$ 1 ), and we know the minimum number of positive, negative and idle entries in $\mathbf{S}^{(T)}$. Consequently, we have $\left(N-\eta_{l i}\right)+$ $\left(N-\eta_{l i}\right)+\eta_{l i} \leq N$ i.e. $N \leq \eta_{l i}$. This leads to a contradiction since $N \geq 2 \eta_{l i}>\eta_{l i}$. It follows that $T \geq 2$.

Let us consider a node $k$ at the other end of the network (opposite to the final destination end), where $1 \leq k \leq \eta_{l i}$. $k$ transmits at least one message to its neighbor $k+\eta_{l i}$. In turn, node $k+\eta_{l i}$ transmits at least one message to $k+2 \eta_{l i}$ at time slot $t^{\prime}$. During $t^{\prime}-1, k$ cannot transmit to avoid interference at $k+2 \eta_{l i}$ i.e. $k$ remains idle. As a result, there are at least $\eta_{l i}$ additional idle entries in $\mathbf{S}^{(T)} \forall T$. Furthermore, we look for maximizing the throughput knowing that among the $N \times T$ entries of $\mathbf{S}^{(T)}$, there are necessarily $N-\eta_{l i}$ positive entries, $N-\eta_{l i}$ negative entries and $2 \eta_{l i}$ idle entries, and the period is such that $T \geq 2$. As long as every $\mathbf{S}^{(T)}$ contains at least $2 N=$ $N-\eta_{l i}+N-\eta_{l i}+2 \eta_{l i}$ entries, the problem of maximizing the throughput can be reduced to minimizing the period $T$, whose minimum value is 2 . Therefore, $Y \leq\left(\left(N-\eta_{l i}\right) / 2\right)$.

$$
\mathbf{S}^{(4)}=\left[\begin{array}{cccc}
4 & 4 & 0 & 0 \\
0 & 0 & 5 & 5 \\
6 & 6 & 0 & 0 \\
7 & -1 & -1 & 7 \\
-2 & 8 & 8 & -2 \\
9 & -3 & -3 & 9 \\
-4 & -4 & 10 & 10 \\
11 & 11 & -5 & -5 \\
-6 & -6 & 12 & 12 \\
-7 & 0 & 0 & -7 \\
0 & -8 & -8 & 0 \\
-9 & 0 & 0 & -9
\end{array}\right]
$$

\section{TRANSMISSION SCHEDULE ACHIEVING THE UPPER BOUND}

After deriving the upper bound on network throughput, we investigate schedules that achieve this limit. For illustration purposes, we propose the schedule $\mathbf{S}^{(4)}$ for the regular 3-line 
grid 12-node network with multihop relaying $(N=12$ and $\eta_{l i}=3$ ). It is a very representative example. By exploiting propagation delays to favor, on one hand, as many concurrent transmissions as possible, and on the other hand, to concentrate interference at unintended nodes, one can maximize the network throughput. For instance, in S(4), the interference from the neighbors 4,6 and 8 arrives at node 5 during time slot 2 , when it is transmitting. $\mathbf{S}^{(4)}$ achieves the upper bound of $((12-3) / 2)=4.5$.

When formulating generally, over four time slots, each first node on odd lines transmits successively two messages and remains idle during the other two time slots. However, each first node on even lines remains idle during two time slots and transmits in turn two messages consecutively. The other nodes receive consequently from their respective downstream neighbors during two time slots, and transmit two messages to their respective upstream neighbors during two time slots, until they reach the final destination nodes. The resulting schedule contains $2\left(N-\eta_{l i}\right)$ transmissions over a period $T=4$. Hence, the network throughput is $Y=\left(\left(N-\eta_{l i}\right) / 2\right)$. In fact, such a design is obtained using appropriate problem formulation and solution method.

As in [9], we formulate the problem of finding a $T$-periodic optimal schedule, denoted by $\mathbf{S}^{*}$, as a sequential decision problem. Due to the lack of space, we simply indicate the main features of the method. The current state of the system is known and represented by $\mathbf{S}^{\{t\}}$, which is the partial schedule given all transmissions occurring between time slots $t-2 g$ and $t-1$. Note that a transmission will not remain in the network more than $2 g$ time slots. $\mathbf{x}^{\{t\}}$ denotes the action (transmission decision) taken at $t$. Moreover, we use a numbering scale designated by $a$ within each time slot $t$. Let $H^{\{t\}}$ denote the number of transmissions in time slot $t$. After $a-1$ transmission decisions and using the transition function $\Delta($.$) , the partial$ schedule $\dot{\mathbf{S}}^{\{t, a\}}$ is combined with the transmission decision $\dot{\mathbf{x}}^{\{t, a\}}$ to find the next partial schedule, as indicated by

$$
\begin{aligned}
& \dot{\mathbf{S}}^{\{t, a+1\}}=\Delta\left(\dot{\mathbf{S}}^{\{t, a\}}, \dot{\mathbf{x}}^{\{t, a\}}\right) \quad \forall a<H^{\{t\}}, \\
& \dot{\mathbf{S}}^{\{t+1,1\}}=\Delta\left(\dot{\mathbf{S}}^{\left\{t, H^{\{t\}}\right\}}, \dot{\mathbf{x}}^{\left\{t, H^{\{t\}}\right\}}\right) .
\end{aligned}
$$

Since we do not know the true action value function, it should be estimated iteratively at each time slot. To achieve this, standard algorithms require exhaustive state space and decision space enumerations to be performed. Therefore, the computation becomes prohibitively expensive.

$$
C_{k l \delta}\left(\dot{\mathbf{S}}^{\{t\}}\right)=\left\{\begin{array}{ccc}
0, & \text { if } \quad D_{k l}>g \\
0, & \text { if } l=k \\
0, & \text { if } \quad \dot{\mathbf{S}}_{k, t^{\prime}}^{\{t\}} \neq 0 \\
0, & \text { if } \quad \dot{\mathbf{S}}_{l, t^{\prime}+D_{k l}}^{\{t\}} \neq 0 \\
0, & \text { if } \exists i \text { s.t. }\left[D_{i l} \leq 2 g\right] \& \\
& {\left[\dot{\mathbf{S}}_{i, t^{\prime}+D_{k l}-D_{i l}}^{\{t\}}>0\right]} \\
0, & \text { if } \exists i, j \text { s.t. }\left[D_{k j} \leq 2 g\right] \& \\
& {\left[D_{i j} \leq g\right] \&\left[\dot{\mathbf{S}}_{i, t^{\prime}+D_{k j}-D_{i j}}^{\{t\}}=j\right]} \\
1, & \text { otherwise. }
\end{array}\right.
$$

Approximation of the action value function is a more practical technique. This method is based on the concept of approximate dynamic programming [12]. Given a transmission decision $\dot{\mathbf{x}}$, the capacity of a state to accommodate future transmissions within $2 g$ time slots is used as an approximate measure of the state-action pair value. We use the transmission indicator function $C_{k l \delta}$ to determine if a transmission from node $k$ to node $l$ at $t^{\prime} \geq t$ is allowed, given the partial schedule $\dot{\mathbf{S}}^{\{t\}}$ at $t$. In the expression of $C_{k l \delta}$ provided above $\delta=t^{\prime}-t$. by

Thus, the action value function approximation $\dot{Q}$ is given

$$
\dot{Q}\left(\Delta\left(\dot{\mathbf{S}}^{\{t\}}, \dot{\mathbf{x}}\right), \dot{\mathbf{x}}^{\prime}\right)=\sum_{k=1}^{N} \sum_{l=1}^{N} \sum_{\delta=0}^{2 g} C_{k l \delta}\left(\Delta\left(\dot{\mathbf{S}}^{\{t\}}, \dot{\mathbf{x}}\right)\right)
$$

where $\dot{\mathbf{x}}^{\prime}$ is the decision immediately following $\dot{\mathbf{x}}$. Note that only decision $\dot{\mathrm{x}}$ matters in the optimal action finding process, unlike $\dot{\mathbf{x}}^{\prime}$. Furthermore, we update the decision space $\dot{\mathcal{X}}$ to $\ddot{\mathcal{X}}$ before making use of the action value function, in order to orient all transmissions in one direction

$$
\ddot{\mathcal{X}}=\{(i, j) \in \dot{\mathcal{X}} \quad \text { s.t. } \quad i<j\} .
$$

Algorithm 1 summarizes the above discussed steps.

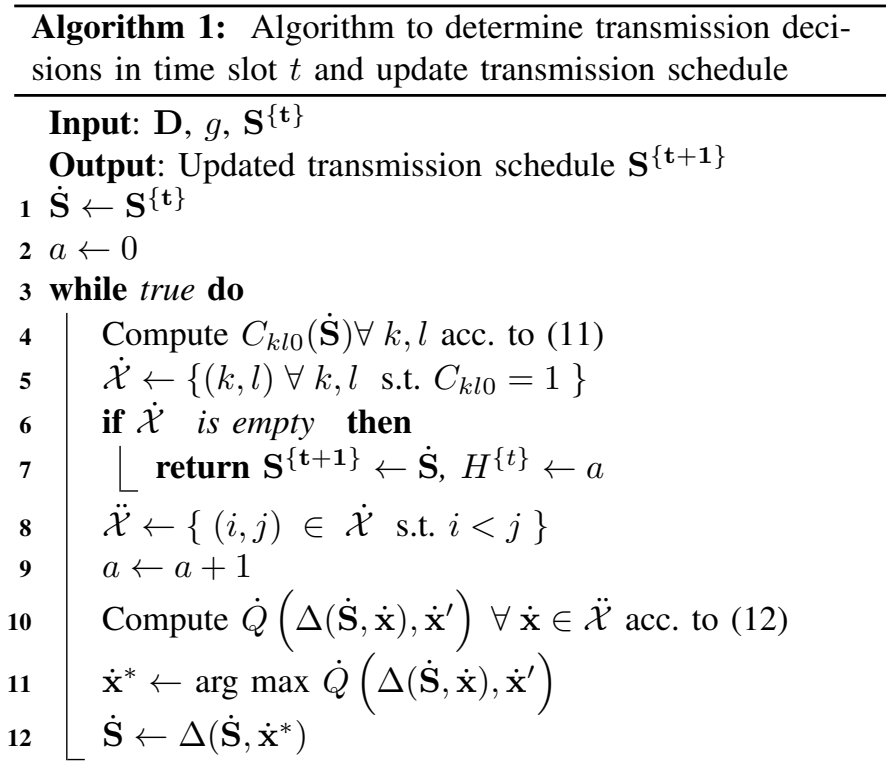

\section{CONCLUSION}

This paper considers TDMA-based MAC protocols which take advantage of large propagation delays to perform maximum network throughput in regular multiline grid networks with multihop relaying. We derive the upper bound and propose optimal schedules obtained using appropriate algorithm. This study provides substantial results with regards to multiline grid topology with multihop relaying. However, in order to understand at a fundamental level how advantageous nonzero delays are in such geometries, it would be worthwhile to explore the linear topology with respect to traffic policy, collision domain extent, and various fairness constraints. 


\section{ACKNOWLEDGMENT}

This work has been supported in part by the "Fonds unique interministériel" (FUI) France within the COMET project.

\section{REFERENCES}

[1] J. Heidemann, M. Stojanovic, and M. Zorzi, "Underwater Sensor Networks: Applications, Advances, and Challenges," Philosophical Transactions of the Royal Society (A), pp. 158-175, Jan. 2012.

[2] S.C. Ergen and P. Varaiya, "PEDAMACS: Power Efficient and Delay Aware Medium Access Protocol for Sensor Networks," IEEE Transactions on Mobile Computing, vol. 5, no. 7, pp. 920-930, July 2006.

[3] B. Peleato and M. Stojanovic, "Distance Aware Collision Avoidance Protocol for Ad-hoc Underwater Acoustic Sensor Networks," IEEE Communications Letters, vol. 11, no. 12, pp. 1025-1027, Dec. 2007.

[4] Xiaoxing Guo, M.R. Frater, and M.J. Ryan, "Design of a PropagationDelay-Tolerant MAC Protocol for Underwater Acoustic Sensor Networks," IEEE Journal of Oceanic Engineering, vol. 34, no. 2, pp. 170 180, April 2009.

[5] K. Kredo, P. Djukic, and P. Mohapatra, "STUMP: Exploiting Position Diversity in the Staggered TDMA Underwater MAC Protocol," in Proc. IEEE INFOCOM 2009, Rio de Janeiro, Brazil, Apr. 2009.

[6] Hai-Heng Ng, Wee-Seng Soh, and M. Motani, "A BidirectionalConcurrent MAC Protocol With Packet Bursting for Underwater Acoustic Networks," IEEE Journal of Oceanic Engineering, vol. 38, no. 3, pp. 547-565, July 2013.

[7] Wenyi Zhang, M. Stojanovic, and U. Mitra, "Analysis of a Linear Multihop Underwater Acoustic Network," IEEE Journal of Oceanic Engineering, vol. 35, no. 4, pp. 961-970, Oct. 2010.

[8] Yang Xiao, Miao Peng, J. Gibson, G.G. Xie, Ding-Zhu Du, and A.V. Vasilakos, "Tight Performance Bounds of Multihop Fair Access for MAC Protocols in Wireless Sensor Networks and Underwater Sensor Networks," IEEE Transactions on Mobile Computing, vol. 11, no. 10, pp. 1538-1554, Oct. 2012.

[9] M. Chitre, M. Motani, and S. Shahabudeen, "Throughput of Networks With Large Propagation Delays," IEEE Journal of Oceanic Engineering, vol. 37, no. 4, pp. 645-658, Oct. 2012.

[10] D. Jing, L. Ben, and P.K. Varshney, "Tuning the Carrier Sensing Range of IEEE 802.11 MAC," in Proc. IEEE Global Telecommunications Conf., Dallas, Texas USA, Nov. 2004.

[11] S. Boppana and J.M. Shea, "Overlapped Carrier-Sense Multiple Access (OCSMA) in Wireless Ad Hoc Networks," IEEE Transactions on Mobile Computing, vol. 8, no. 3, pp. 369-383, Mar. 2009.

[12] Warren B. Powell, Approximate Dynamic Programming: Solving the Curses of Dimensionality, Wiley-Interscience, New York, 2007. 